\title{
Presentation
}

\section{Population Based Cohort Study for Pediatric Infectious Diseases Research in Vietnam}

\author{
Lay-Myint Yoshida ${ }^{1 *}$, Motoi Suzuki ${ }^{1}$, Vu Dinh Thiem², Wolf Peter Smith ${ }^{3}$, Ataru Tsuzuki ${ }^{1}$, Vu Thi Thu Huong ${ }^{2}$, \\ Kensuke Takahashi ${ }^{1}$, Masami Miyakawa ${ }^{4}$, Nguyen Thi Hien $\mathrm{Anh}^{2}$, Kiwao Watanabe ${ }^{1}$, Nguyen Thu Thuy Ai ${ }^{5}$, \\ Le Huu Tho ${ }^{6}$, Paul Kilgore ${ }^{7}$, Hiroshi Yoshino ${ }^{1}$, Michiko Toizumi ${ }^{1}$, Michio Yasunami ${ }^{1}$, Hiroyuki Moriuchi ${ }^{4}$, \\ Dang Duc Anh ${ }^{2}$ and Koya Ariyoshi ${ }^{1}$ \\ Published online 24 May, 2014
}

\begin{abstract}
A population-based cohort study on pediatric infectious diseases was established at Khanh Hoa Province, central Vietnam in 2006, to determine the etiology and risk factors for severe pediatric infectious diseases (SPID) such as acute respiratory infection (ARI), diarrhea and dengue which are the major causes of under 5 mortality. A population census survey was conducted in Nha-Trang and Ninh-Hoa to collect demographic, social-behavioral data and disease burden on SPID. The study site covered a population of 353,525 residing in 75,826 households with 24,781 children less than 5 years. Hospital databases from two hospitals covering the region were obtained. Linking the census and hospital databases, we were able to investigate on a variety of SPID such as environmental tobacco smoking exposure and increased risked of pediatric pneumonia hospitalization, population density, water supply and risk of dengue fever and animal livestock and risk of hospitalized diarrhea. To determine incidence, viral etiology and risk factors for pediatric ARI/pneumonia, we setup a population based prospective hospitalized Pediatric ARI surveillance at Khanh Hoa General Hospital, Nha-Trang in February 2007. The study has revealed RSV, rhinovirus and influenza A as major viral pathogens, role of multiple viral infection and its interaction with bacteria in the development of pneumonia. In addition, we are also conducting a birth cohort study to investigate the incidence of congenital infection and its impact on physical-neurological development, and role of host genetic polymorphism on SPID hospitalization in Vietnam. Population mobility, high cost of regular census update and low mortality are the challenges.
\end{abstract}

Key words: Population based cohort, pediatric, infectious diseases, Vietnam

\section{INTRODUCTION}

\section{Tetsu Yamashiro}

We need to proceed to the next speaker. Dr. LayMyint Yoshida. He belongs to Department of Pediatric Infectious Diseases, Institute of Tropical Medicine, Nagasaki University. He has been conducting a very important epidemiological research work in Vietnam since the beginning of this J-GRID program. His title is "Population Based Cohort Study for Pediatric Infectious Diseases

\section{Research in Vietnam."}

\section{Lay-Myint Yoshida}

Thank you very much for your nice introduction. I'm from the Department of Pediatric Infectious Diseases, and my department is very new. Actually, originally I was from the Clinical Medicine Department, Institute of Tropical Medicine, known as NEKKEN NAIKA, but it was recently expanded into the NEKKEN NAIKA, Pediatric Infectious Diseases Department and Clinical Development Department. Then I moved to Pediatric Infectious Diseases

\footnotetext{
${ }^{1}$ Institute of Tropical Medicine, Nagasaki University, 1-12-4, Sakamoto, Nagasaki 852-8523, Japan

${ }^{2}$ National Institute of Hygiene and Epidemiology, No 1, Yersin street, Hanoi, Vietnam

${ }^{3}$ London School of Tropical Medicine and Hygiene, Keppel St, Bloomsbury, London WC1E 7HT, United Kingdom

${ }^{4}$ Department of Pediatrics, Nagasaki University Hospital, Graduate School of Biomedical Sciences, Nagasaki University, 1-12-4, Sakamoto, Nagasaki 852-8523, Japan

${ }^{5}$ Khanh Hoa General Hospital, 19 Yersin Street Nha Trang, Vietnam

${ }^{6}$ KhanhHoa Provincial Public Health Service, 3A Han Thuyen Street, Nha Trang City, Khanh Hoa Province, Vietnam

${ }^{7}$ College of Pharmacy and Health Sciences, Wayne State University, 259 Mack Ave. Suite 2118, Detroit, MI 48201

*Corresponding author:

Tel: +81 (0)95-819-7284

Fax: +81 (0)95-819-7843

E-mail:1myoshi@nagasaki-u.ac.jp
} 
Department. But basically, we're working together. I'm responsible for working for the clinical research group of our Nagasaki-Vietnam collaborative research project.

The main objectives of our study in Vietnam is to determine the etiology and risk factors for severe common pediatric infectious diseases (SPID); acute respiratory infection/pneumonia, diarrhea which are the major causes of under 5 mortality and dengue haemorrhagic fever (DHF) which is also a leading cause of serious illness and death among children in some Asian countries. It has been reported that an estimated 500,000 people with DHF require hospitalization each year, a very large proportion of whom are children and about $2.5 \%$ of those affected die (UNICEF. The state of world's children 2008. http:// www.unicef.org/sowc08/docs/sowc08.pdf.), (http://www. who.int/mediacentre/factsheets/fs117/en/) [1].

Our research program is funded by the Japan Initiative for Global Research Network on Infectious Diseases (J-GRID) and our main counterpart in Vietnam is National Institute of Hygiene and Epidemiology (NIHE). NIHE is one of the very prominent internationally recognized research institutes in the Asia and we are very fortunate to have a chance to work together with our NIHE counterparts, which have a lot of international research experience. Today, I'm going to present about our success and some challenges that we are facing in conducting this study.

We, NEKKEN together with NIHE received the funding from J-GRID in 2005 and started the study in 2006. First, we selected the study site to be conducted in Nha Trang city located in the central part of Vietnam. One of the reasons is NIHE has a very strong collaboration with Khanh Hoa Health Service Department in Nha Trang. By collaborating with the Khanh Hoa Health Service Department, we could obtain access to the hospital and also the community/commune health centers. We also have collaboration with the local research institute in Nha Trang like Nha Trang Pasteur Institute. Working together in this network (Fig. 1), we established a population based level of infectious disease research surveillance study in Nha Trang.

We wanted to study important health issues in terms of incidence, not the prevalence, so we needed to have population denominators. Nha Trang is located in the central eastern part of Vietnam (Fig. 2). Geographically, Nha Trang is a unique place. You can see in the below figure that the sea in the eastern side. The west, north, and south are surrounded by mountains. The population is living mainly in the center area and there is only one hospital, Khanh Hoa General Hospital (KHGH) which is providing hospital care in this area. This gives us an idea that if we have a population data and good health disease burden data

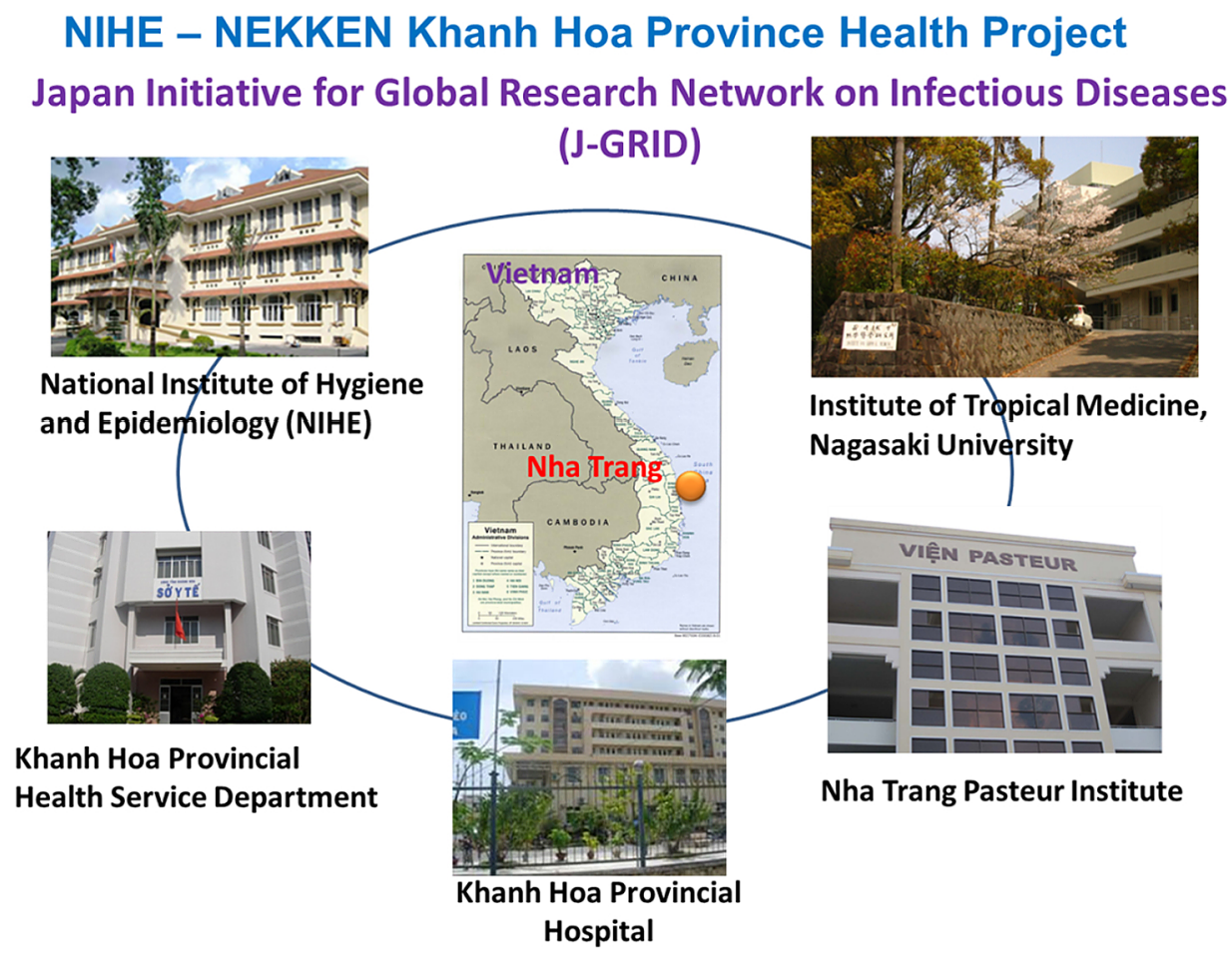

Fig. 1. Research collaborative network. 


\title{
Establishment of Clinical Epidemiological Field in Khanh Hoa Province Nha Trang city \& Ninh Hoa district \\ 75,828 household, population: 353,525 with 24,781 children less than $5 y r s$
}

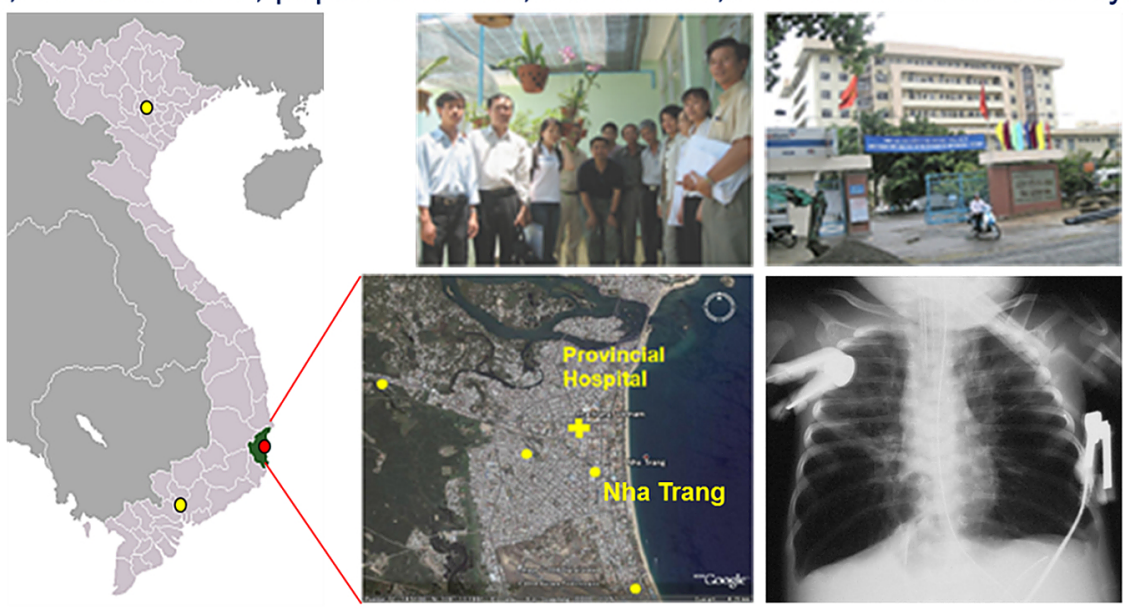

\begin{abstract}
A population based cohort study on Pediatric Infectious Diseases at Khanh Hoa Province, central Vietnam was established in 2006

$>$ Hospital data bases were acquired form Khanh Hoa General hospital and Ninh Hoa District hospital the two hospitals covering the target population $\rightarrow$ linked to population study database.
\end{abstract}

Fig. 2. Establishment of population based cohort study in central Vietnam.

from the hospital and the community, by linking the community data together with this hospital data, we should be able to answer many important research questions happening in this region and in Vietnam.

First, we need population based data, so in the beginning of the study in 2006, we conducted a census survey in Nha Trang city and Ninh Hoa district covering over 75,000 household with the population of over 350,000 which included nearly 25,000 children less than 5 years [2].

When we conducted the population census survey, we not only collected the population data, but we asked for their behavioral information such as health-seeking behavior, environmental issues such as smoking or what kind of animals they are breeding or whether they use bed net or not. We also collected the disease burden and healthseeking behavior information such as if the child gets ARI, what do you do? Do you go to the polyclinic, do you go to the private clinic, do you go to the hospital or do you just buy some drugs and stay at home or you just use traditional medicine? By asking those questions, it gives us an idea that how much proportion of the population is actually going to the hospital and what was happening in the community. We did the population census and disease burden survey, and obtained the hospital database from KHGH. Vietnam introduced digitalized hospital database system in 2003. Therefore we could obtain ICD-10 coded database from the hospital in this region. After we conducted this census survey, we were able to link the two databases and then analyzed it to address different health-related research questions.

In this presentation, I'm going to give a few example of what kind of research studies we conducted in three different aspects; behavior and environmental, etiological, and congenital and host genetic factors.

\section{BEHAVIOR AND ENVIRONMENTAL ISSUE RELATED STUDIES}

Smoking and pneumonia: Does environmental tobacco smoking exposure increase the risk of pediatric pneumonia hospitalization?

Smoking is very common in Vietnam therefore we were interested in the association of pediatric infectious diseases like pneumonia and smoking. To address this issue we analyzed the information on demographics, socioeconomic status and house environment, including smoking status of each household member that we collected during our population-based large-scale cross-sectional survey which covered all residents of 33 communes in Nha Trang city and Ninh Hoa distirct, in central Vietnam. Hospital admissions for pneumonia among children aged less than 5 years in each household in the previous 12 
months were also recorded based on caregiver's report for the analysis.

The result of the study indicate that a total of 353,525 individuals were living in 75,828 households in the study areas. Of these, $24,781(7.0 \%)$ were less than 5 years. The prevalence of environmental tobacco smoking (ETS) was $70.5 \%$ and the period prevalence of hospital admissions for pneumonia was $2.6 \%$. Multiple logistic regression analysis showed that exposure to ETS was independently associated with hospital admissions for pneumonia (adjusted odds ratio $1.55,95 \%$ CI 1.25 to 1.92 ). The prevalence of tobacco smoking was higher among men than women (51.5\% vs $1.5 \%)$. It is estimated that $28.7 \%$ of childhood pneumonia in this community is attributable to ETS.

Therefore we concluded that children in Vietnam are exposed to substantial levels of ETS which results in 44,000 excess hospital admissions due to pneumonia each year among children aged 5 years $[3,4]$.

\section{Bed net usage and Dengue: Can daytime use of bed nets not treated with insecticide reduce the risk of dengue hemorrhagic fever hospitalization among children in Vietnam?}

Next topic is dengue. Because we have household behavior information related to daytime bed net usage and DHF hospitalization data, we investigated the effect of daytime bed net use on preventing dengue hemorrhagic fever (DHF) hospitalization among children in Vietnam. We utilized our population-based cross-sectional survey data and conducted case-control study in Khanh Hoa Province. In Vietnam there is a custom that some preschoolchildren and school children take a nap during lunch break prior to returning to school. In our study population, out of 36,901 children 2-10 years of age, most used untreated bed nets during the night (98.3\%) compared with $8.4 \%$ during the day. The results of the case-control study (151 cases who were hospitalized with DHF in the provincial hospitals and 604 age-matched neighborhood controls), illustrated that there was no difference in DHF hospitalization between the two groups (adjusted odds ratio $=0.56,95 \% \mathrm{CI}=0.23-1.39)[5]$.

When we checked the association of bet net usage and malaria infection rate, the use of bed net without any insecticide treated one, won't be affected. This is similar to what we are observing with DHF in our study. If the people have used insecticide impregnated bed net, it might reduce the dengue infection and hospitalization too. Further studies are needed to confirm this finding.

\section{Water supply and Dengue: Optimal population density, effect of water supply, and the risk of dengue fever in Vietnam: cohort study and spatial analysis}

The next topic related to environmental issue is the relation of water supply and dengue. This study was conducted in collaboration with an epidemiologist, a research fellow from London School of Tropical Medicine and Hygiene who visited our institute as a JSPS research fellow.

The major vector of dengue viruses (Aedes aegypti), often breeds in water storage containers used by households without tap water supply. We analyzed the interaction between human population density and lack of tap water as a cause of dengue fever outbreaks with the aim of identifying geographic areas at highest risk. An individuallevel cohort study in a population of 75,000 geo-referenced households in Vietnam over the course of two epidemics, on the basis of dengue hospital admissions $(n=3,013)$ was studied. Space-time scan statistics and mathematical models were applied to confirm the findings.

Surprisingly a narrow range of critical human population densities between around 3,000 to 7,000 people $/ \mathrm{km}^{2}$ prone to dengue outbreaks were identified (Fig. 3). In the study area, this population density was typical of villages and some peri-urban areas. Scan statistics showed that areas with a high population density or adequate water supply did not experience severe outbreaks. The risk of dengue was higher in rural compared to urban areas, largely explained by lack of piped water supply, and in human population densities more often falling within the critical range. Mathematical modeling suggests that simple assumptions regarding area-level vector/host ratios may explain the occurrence of outbreaks. Thus we concluded from this study that improving water supply and vector control in areas with a human population density critical for dengue transmission could increase the efficiency of dengue control efforts. This was a very unique analysis and published in PLOS Medicine [6] which is a very good journal.

\section{Animal and Diarrhea: Animal livestock and risk of hospitalized diarrhea in Vietnam}

Next we investigated another potential behavior or environmental risk factor that may be related to diarrhea. In Nha Trang Vietnam, a lot of household has animals; chicken, stocks, pigs etc., in their household. The animals are coming in and out of the house which may affect the hygiene of the household members. To investigate this potential risk factor, we investigated whether breeding animal in the household increased incidence of the diarrhea hospitalization. 


\section{Water supply and Dengue} Optimal population density, effect of water supply, and the risk of dengue fever in Vietnam: cohort study and spatial analysis

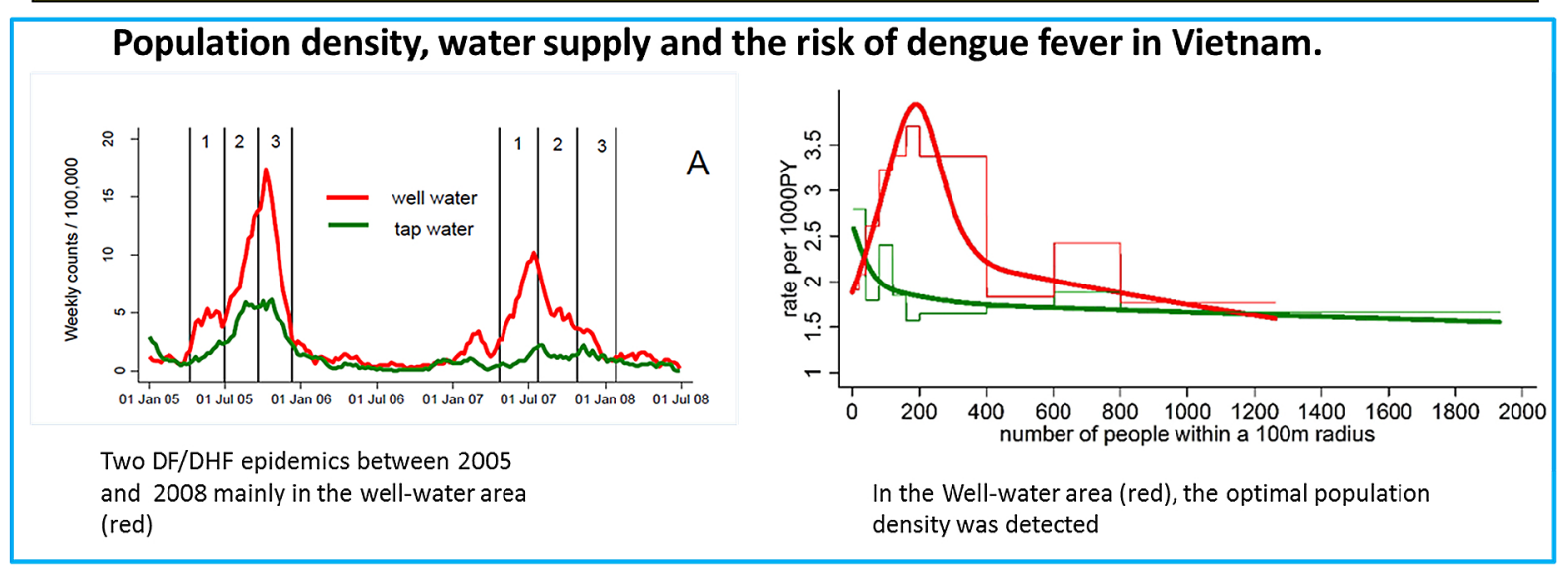

- Critical population densities prone to dengue outbreaks: 3,000-7,000 people/km²

- Risk of dengue: higher in rural, largely explained by lack of water supply

- Improving water supply and vector control in areas with a population density critical for dengue transmission could increase the efficiency of control efforts.

Schmidt WP, Suzuki M, Thiem VD, White RG, Tsuzuki A, Yoshida $L M$, Yanai $H$, Haque $U$, Tho le $H$, Anh DD, Ariyoshi K. PLoS Med. 2011 Aug;8(8):e1001082

Fig. 3. Optimal population density, effect of water supply, and the risk of dengue fever in Vietnam.

Our population-based cohort of 353,525 individuals, living in 75,828 households in Khanh Hoa Province, Vietnam, with baseline data covering geo-referenced information on demography, socioeconomic status and household animals was investigated. Geographic information system (GIS) was applied to calculate the density of livestock. The data were linked to hospitalized diarrhea cases of children less than 5 years recorded at two hospitals treating patients from the area as inpatients in the study area (Fig. 4).

Overall, 3,116 children with diarrhea were hospitalized during the study period. The incidence of diarrhea hospitalization in the study area was 60.8/1000 child-years. Male gender, age $<2$ years, higher number of household members and lack of tap water were significantly associated with an increased risk of diarrhea. However there was no evidence that ownership of livestock increased the risk of diarrhea.

This was published in Tropical Medicine \& International Health and one of our Vietnamese Ph.D. students did this paper [7].

\section{Active Surveillance Studies}

The studies that I have introduced above were based on large census database from our population survey linking to the hospital database. In 2007, to enhance our research capacity, we established active surveillance studies to collect additional information which were not available in the hospital in the study area.

\section{Acute Respiratory Infection Surveillance}

Acute respiratory tract infections (ARI) are a major cause of morbidity and mortality in children worldwide. Viruses are common causes of lower respiratory tract disease in children and represent a major public health problem. Due to the limited resources and facilities for virus isolation and detection, the role of viral respiratory pathogens in Vietnam has not been well-studied. Therefore to investigate the magnitude of viral respiratory infections among hospitalized children in Vietnam, we established a population based active pediatric ARI surveillance study and applied a newly developed multiplex-polymerase 


\section{Animal and Diarrhea Animal livestock and risk of hospitalized diarrhea in Vietnam}

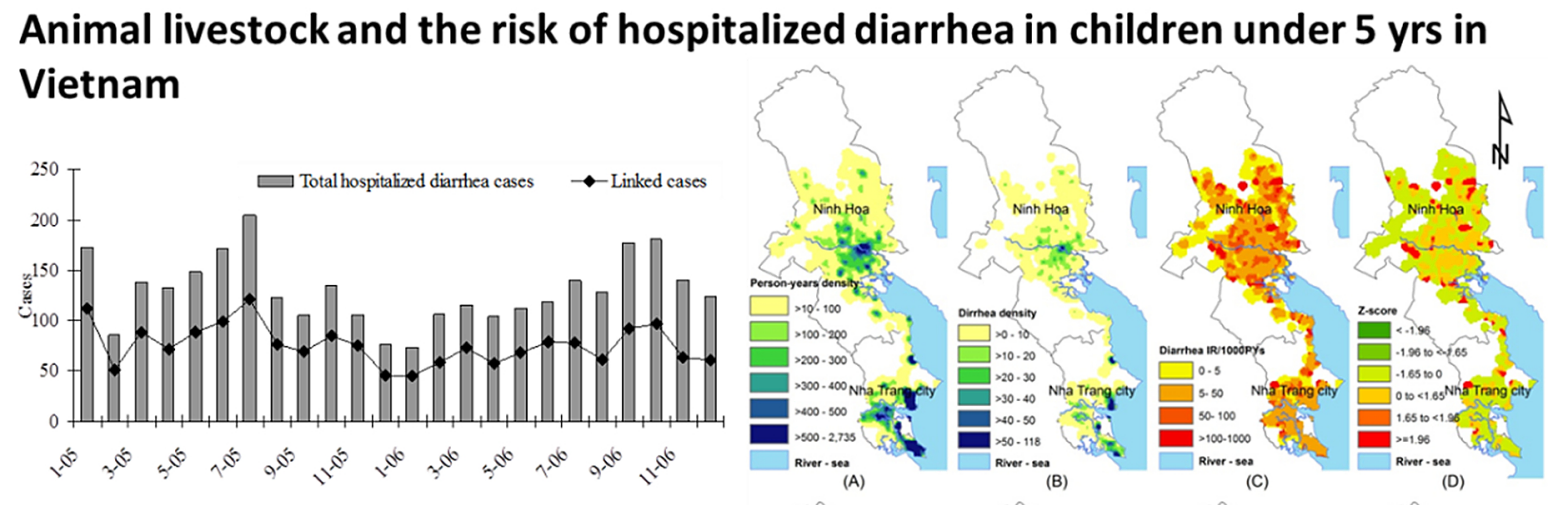

-3116 children with diarrhea hospitalization - Incidence:60.8/1000 child-yr -Increased risk: Male, age < 2 years old, higher no. of household members, lack of tap water

\section{There was no evidence that ownership of livestock increased the risk of diarrhea.}

Vu Dinh Thiem, et,al, Trop Med and Int Health, 2012 May;17(5):613-21

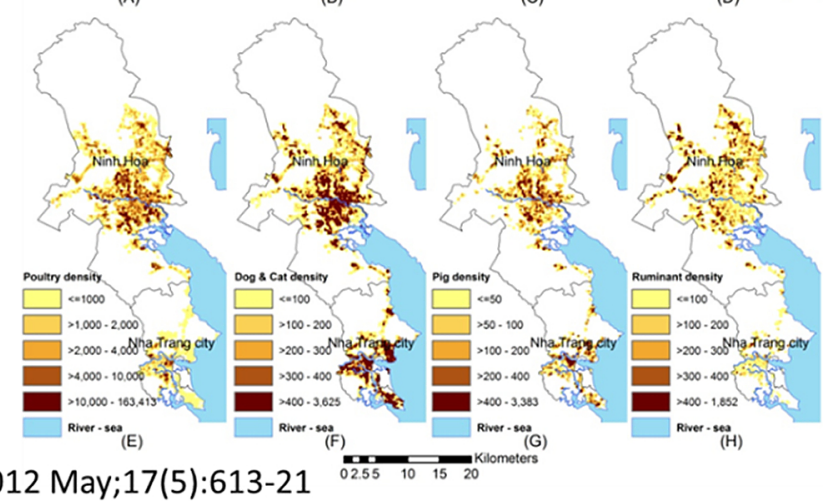

Fig. 4. Animal livestock and risk of diarrhea in Vietnam.

chain reaction (PCR) technique that can detect 13 different respiratory viruses, in central Vietnam.

As previous speaker Professor Oshitani pointed out, even though blood culture examination was performed in the hospital, the positive rate was usually very low. Similarly, in our invasive pneumococcal study, we conducted blood culture for about 1000 samples however we obtained only about 7 or 8 positive result that means positive rate of less than $1 \%$ [8]. It was difficult to interpret the etiology based on the limited blood culture result. High rate $60 \%$ or $70 \%$ of antibiotic usage before coming to the hospital may be the most likely reason for this low blood culture positive rate. We thought that even the bacteria or the organism died, by using the molecular based methods like PCR, we should be able to amplify the nucleic acid of the pathogen, measure the copy number and it should be related to the disease etiology. Therefore, we set up a multiplex PCR assay to identify 13 different respiratory viruses (influenza A, B, RSV, human metapneumovirus, parainfluenza 1-4, rhinovirus, coronaviruses OC43, 229E, adeno and boca viruses) and 3 different bacterial pathogens including
Streptococcal pneumoniae, Haemophilus influenzae, and Moraxella catarrhalis.

We defined the target population as children living in the 16 communes of Nha Trang City admitted to Khanh Hoa General Hospital (KHGH) which is the only hospital in the region. The study was initiated in 2007 February and is still ongoing. Informed consent was obtained from over 95\% of the eligible ARI cases and enrolled into the study. Clinical epidemiological data were collect very carefully. One Japanese physician was stationed in KHGH to obtain accurate clinical and epidemiological data information, checking the patient and making sure that the data that we are collecting are accurate and correct. Nasopharyngeal swab and blood samples were also collected for viral etiology testing and further testing. Chest x-rays were taken for all the children. To make sure that all the children have a chest $\mathrm{x}$-ray, we pay for the chest $\mathrm{x}$-ray and we collect one copy of it, so that we can review the chest $\mathrm{x}$-ray by ourselves for the data analysis. Detail of the surveillance method was published previously [9]. We have been conducting this ARI surveillance study since 2007 and up to 
now, we have collected over 4,800 samples and we have investigated several research questions.

\section{Viral ARI pathogens and pediatric ARI disease burden in central Vietnam}

A large proportion of these cases are caused by viral pathogens, therefore we investigated the viral pathogens responsible for ARI hospitalization in the region (Fig. 5). Determination of ARI disease burden is also important so we determined the ARI hospitalization incidence in the region using our population census survey data.

Figure 5 shows the incidence rate for 1000 child per year. The data indicated that if there was 1000 children, $144(14.4 \%)$ of them were admitted to the hospital at least once before they became the age of 1 , which was a high disease burden. The abnormal chest X-ray or lower respiratory tract infection rate was about $10 \%$ and radiologically confirmed pneumonia (RCP) cases consisted of about one-third. Main viral pathogens were rhino, RSV and influenza viruses. At least one virus was detected in over $60 \%$ of them. Multiple viral infections were also detected in $12 \%$ of the cases [9].

\section{Streptococcus pneumoniae, viral co-infection and Pneumonia: Association Between Nasopharyngeal Load of Streptococcus pneumoniae, Viral Co-infection, and Radiologically Confirmed Pneumonia in Vietnamese Children}

The next question is about the interaction of the viruses and bacteria in the development of pneumonia or risk of pneumonia? To address this question, in addition to viral testing, bacteria load testing for three typical bacteria pathogens (Streptococcal pneumoniae, Haemophilus influenzae, and Moraxella catarrhalis) were conducted in these samples too. In Figure 6, I'm just showing the $S$. pneumoniae data which had a strong relation with pneumonia, but we also tested and analyzed the data for $H$. influenzae, and M. catarrhalis too. We conducted the data analysis in three groups as a case-control study. We collected the samples from the healthy children as control since healthy children can also carry the bacteria. We included other lower respiratory tract infection and pneumonia cases from our ARI surveillance as cases for the analysis.

If you measure the $S$. pneumoniae bacterial load among these group of children, you can see that the children with the pneumonia have over 1 to 2-log significantly higher bacteria load ( $\mathrm{p}=<0.0001)$ compared to the healthy controls. Interesting thing was if the same child was co-

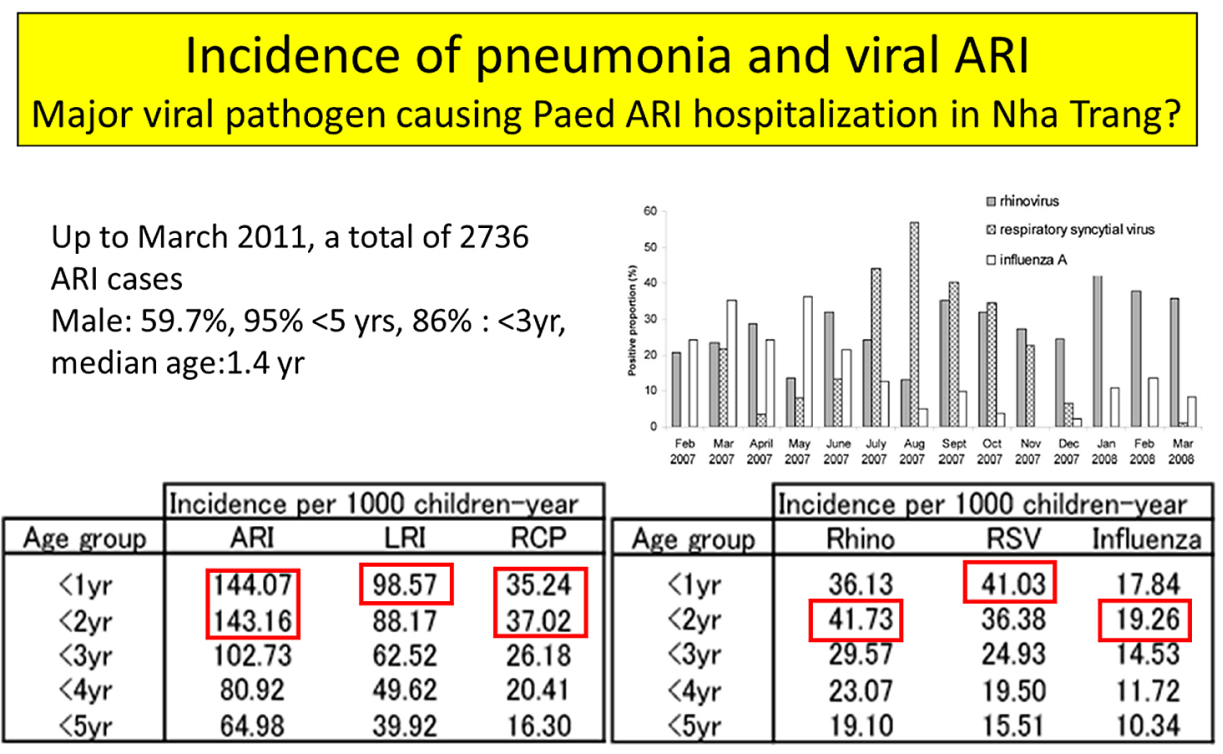

Respiratory viruses: $64 \%$, multiple viral infection: $12 \%$

Rhino, RSV and influenza A viruses were major viral pathogens detected among Pediatric hospitalization

Yoshida LM et, al, Pediatr Infect Dis J. 2010 Jan;29(1):75-7.

Fig. 5. Major viral pathogens causing pediatric ARI hospitalization in central Vietnam. 


\section{S.pneumoniae, viral co-infection and Pneumonia Association Between Nasopharyngeal Load of Streptococcus pneumoniae, Viral Co-infection, and Radiologically Confirmed Pneumonia in Vietnamese Children?}
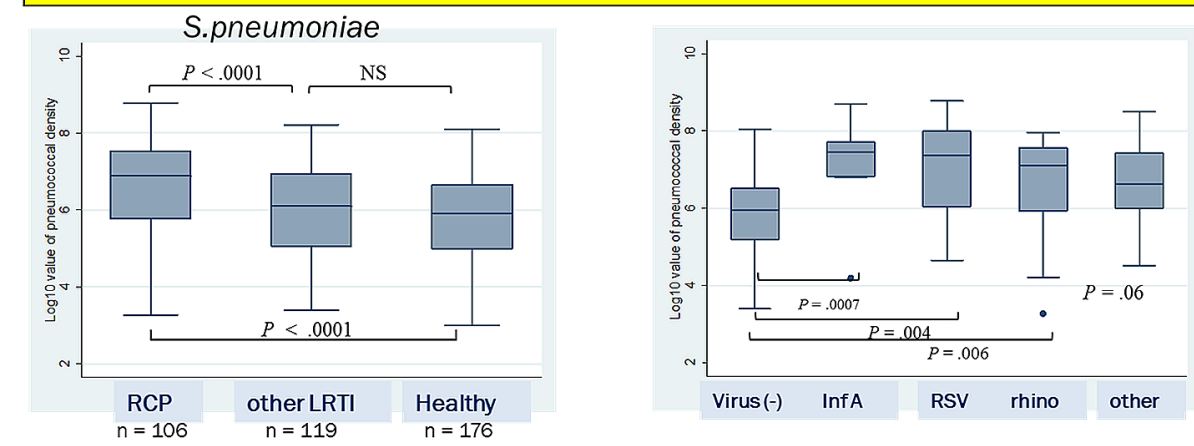

SP load higher in children with RCP compared with healthy controls or other LRTIs

SP load was 15-fold higher in pneumonia children with viral co-infection compared with those without $\left(1.4 \times 10^{7} / \mathrm{ml}\right.$ versus $\left.9.1 \times 10^{5} / \mathrm{ml} ; P=.0001\right) . S P$ load was over 200 -fold higher in serotypeable SP compared with non-typeable SP $\left(2.5 \times 10^{6} / \mathrm{ml}\right.$ versus $\left.1 \times 10^{4} / \mathrm{ml} ; P<.0001\right)$.

Vu THuong et, al, Pediatr Infect Dis J. 2011 Jan;30(1):11-8.

Fig. 6. Association between S. pneumoniae and viral co-infection on risk of penumonia.

infected with another virus, the bacterial loads go up 15 times and then the association with pneumonia further increased significantly ( $\mathrm{p}=<0.005$ ). You can observe this trend clearly in the Figure 6. The median S. pneumoniae bacteria load of virus-negative pneumonia cases, once it was co-infected with other viruses like influenza or RSV, the viral co-infection enhanced the bacterial load significantly and increased the risk of pneumonia in this child [10].

Multiple viral infection and Pneumonia: RSV, its coinfection with other respiratory viruses and increases the risk of pediatric pneumonia hospitalization

Next we moved to the role of multiple viral infections on pediatric pneumonia. Since $12 \%$ of the pediatric ARI cases have multiple viral infection and we asked again what kind of role those multiple viral infections have in the development of pneumonia or disease severity among pediatric ARI cases? We analyze the risk ratio of clinical pneumonia based on single or multiple viral detection results. When RSV co-infection occurred with rhino, parainfluenza or Human metapneumo virus, the risk of pneumonia jumped from 1 to 1.8 or from 1 to even 5 to 6 fold increase with the parainfluenza viral co-infection (Fig. 7). That means the synergistic effect of these viral coinfections caused increase disease severity in these children. But for influenza, it was the opposite effect. When pediatric ARI cases were co-infected with influenza with the other viruses, they did not increase the disease severity [11].

\section{Community based acute respiratory infection/influenza like illness surveillance study}

After talking about the hospital based ARI studies, we were interested in what was happening in the population level too. It was difficult to monitor the whole study population so we selected 1000 households from four communes out of our target 16 communes in Nha Trang. Two polyclinics covering them in the four communes were also included in the study. There were about 5000 people living in the selected 1000 households. We applied a research tool called Healthcare Calendar to collect the information about ARI symptoms like fever, cough, and headache in the community and also what kind of treatment they use. The clinical samples were collected weekly from the ARI cases attending the two polyclinics during the study period.

We have been conducting this study for 33 months and we could identify 3300 ARI/ILI cases from study households. Our study results showed that the ILI incidence per 1000 per month was 22 for the whole population and 71 for less than 5 years. By doing this kind of randomized study, we can also monitor what's happening in the community. The detail analyses are ongoing. 


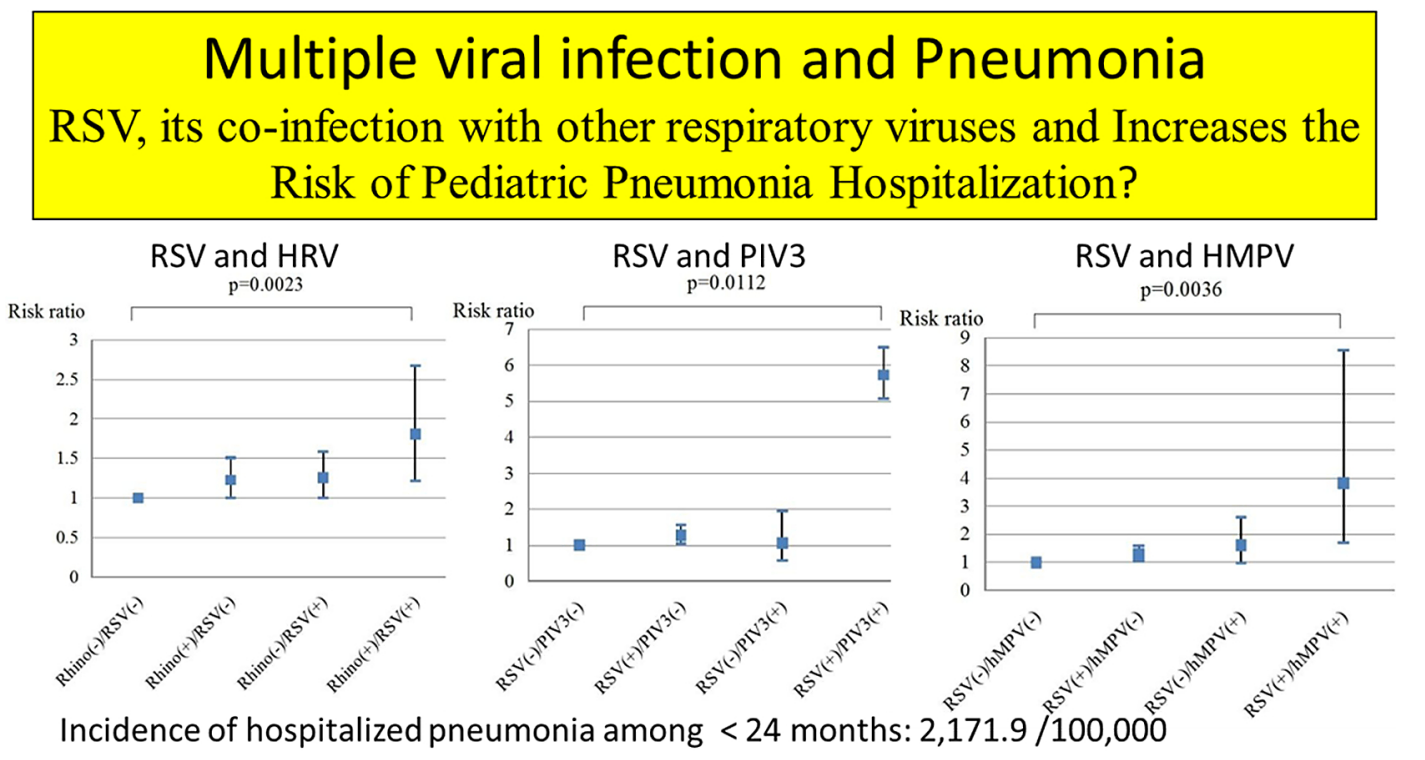

\begin{abstract}
Virus detected in $61 \%$ of ARI cases, RSV: $20.1 \%$, Inf A: $12 \%, 10 \%$ multiple viral infection
RSV and HMPV infections independently increased the risk of pneumonia RSV further increased risk of pneumonia, when co-infected with HRV, HMPV and PIV3 but not with FLUA.
\end{abstract}

Yoshida LM et,al, ERJ, 2013. May

Fig. 7. RSV, its co-infection with other respiratory viruses and Increases the Risk of Pediatric Pneumonia Hospitalization.

\section{Birth cohort study}

We studied about the role of social behavior, environment on infectious diseases like dengue and diarrhea, and viral and bacterial pathogens on pediatric ARI. When you think about the disease pathogenesis, there are always the environmental behavior, pathogen and host gene factors. To cover the scope of the study to all these areas, we established a birth cohort study in Nha Trang, central Vietnam in 2009 (Fig. 8). The main objectives of the study were (i) to investigate the congenital infection and its effect on physical and neurological development of the child, and (ii) to study the role host gene polymorphism on immunological response against vaccine, repeated hospitalization and disease severity in Vietnam.

During the preparation phases of the birth cohort study, we examine the association between gestational weight gain and maternal body mass index (BMI) among Vietnamese women and the risk of delivering an infant too small or too large for gestational age.

To address this issue, a prospective health-facilitybased study of 2,989 pregnant Vietnamese women was conducted in Nha Trang in 2007-2008. Cubic logistic regression was used to investigate the association of interest. Infants were classified into weight-for-gestational-age categories according to weight centiles for the Asian population.

Our study determined that BMI was low $(<18.5)$, normal (18.5-22.9) and high $(\geq 23.0)$ in $26.1 \%, 65.4 \%$ and $8.5 \%$ of the women, in central Vietnam respectively. In each of these BMI categories, the percentage of women who delivered infants too small for gestational age was 18.1, 10.0 and 9.4, respectively, and the mean gestational weight gain was $12.5 \mathrm{~kg}$ (standard deviation, SD: \pm 3.6 ), $12.2 \mathrm{~kg}(\mathrm{SD}: \pm 3.8)$ and $11.5 \mathrm{~kg}(\mathrm{SD}: \pm 4.7)$, respectively. Among women with low BMI, the risk of delivering an infant too small for gestational age ranged from approximately $40 \%$ if the gestational weight gain was $<5 \mathrm{~kg}$ to $20 \%$ if it was $5-10 \mathrm{~kg}$ [12]. Therefore we concluded that having a low BMI, commonly found in Vietnam, puts women at risk of delivering an infant too small for gestational age, especially when total maternal gestational weight gain is $<10 \mathrm{~kg}$.

\section{Risk of congenital rubella infection in Vietnam}

We started the birth cohort study in 2009 and enrolled nearly 2000 mother and newborn babies. We investigated the antibody for rubella among the pregnant mother and found that one third of them did not have rubella antibod- 


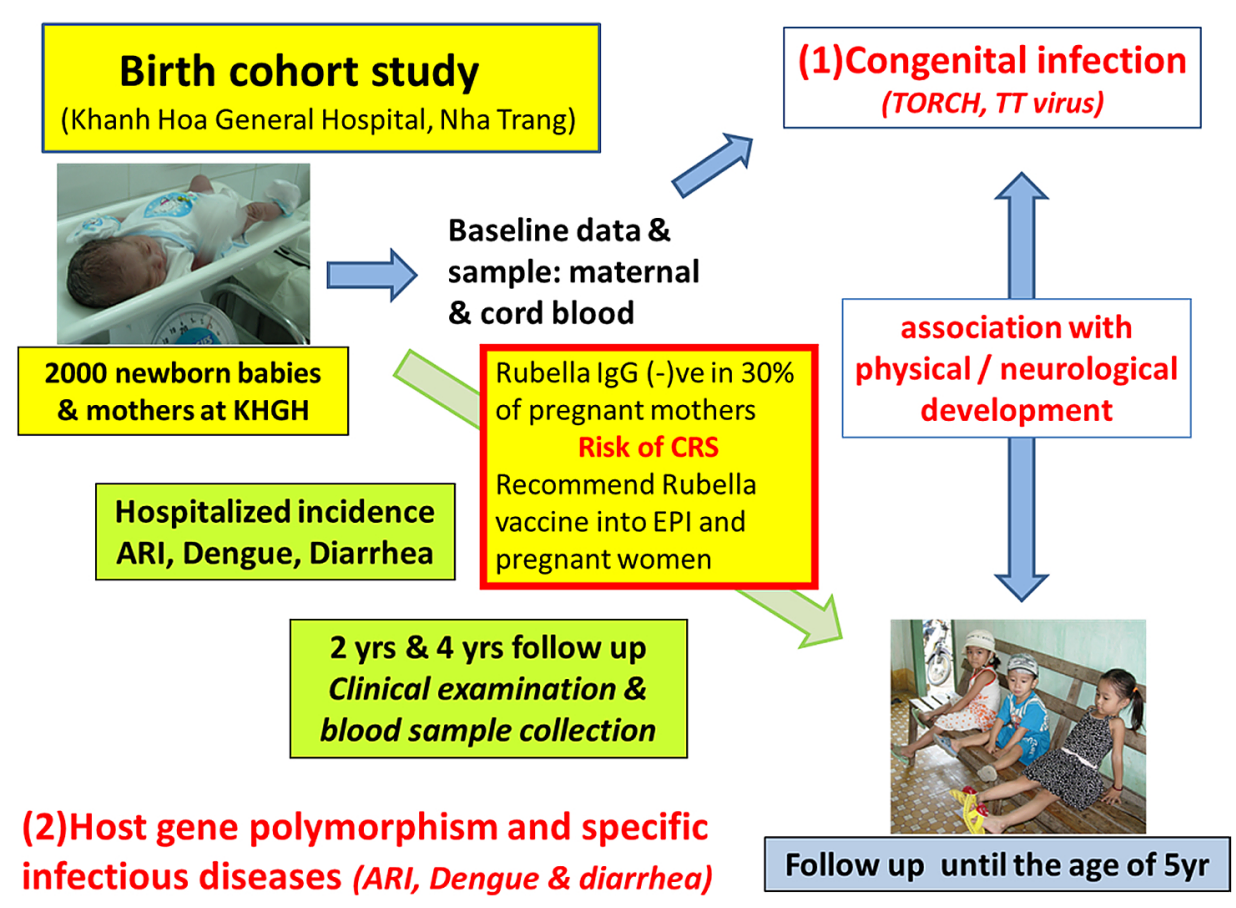

Fig. 8. Establishment of Nha Trang birth cohort study.

\section{Birth cohort: Congenital rubella infection risk Seroprevalence of rubella in the cord blood of pregnant women and congenital rubella incidence in Nha Trang, Vietnam?}

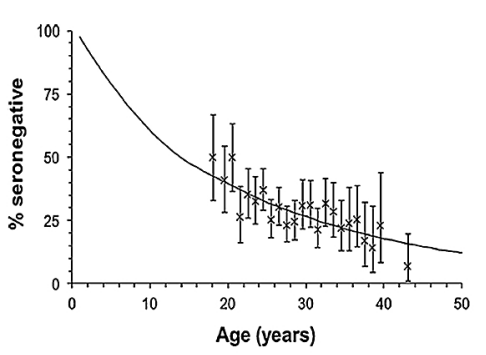

Enrolled: 1988 pairs of mothers aged $17-45$ years and their newborn babies between 2009 and 2010.

Test: Rubella-specific Ig-M and Ig-G were investigated in cord blood samples

Result: 3 in 10 of mothers were susceptive to rubella virus, teenagers at higher risk.

A substantial proportion of women of childbearing age are at risk of rubella infection during pregnancy

This studying finding was used by Vietnam national immunization program to introduce rubella vaccine into EPI in Vietnam!

Miyakawa et.al., Vaccine. 2013, http://www.sciencedirect.com/science/article/pii/S0264410X13011882\#

Fig. 9. Risk of rubella infection among pregnant mothers in Nha Trang, central Vietnam.

ies. This finding showed that there was a risk of congenital rubella infection in central Vietnam and we suggested to the government for rubella vaccine introduction in Vietnam [13]. We also conducted a 2 years follow up study for the birth cohort children in 2011 and successfully follow up nearly $75 \%$ of them. This birth cohort study is ongoing. 


\section{Advantages and disadvantage of working in Vietnam Advantages}

I would like to mention a few advantages that we have compared to the previous presented studies in Philippines or Africa.

(i) The study site selection is very important. If you can select a good study site, you can have advantages of efficiently monitoring the situation.

(ii) Acquisition of skillful staff is also very important. Since Nha Trang field staffs are very well trained and they have experience in conducting various field and hospital based studies, we could achieve a very good outcome.

(iii) Vietnam itself has an excellent up-to-down administration system. In the healthcare system, if you look from the lower level, they have primary healthcare system and then they have polyclinics, they have district hospital and provincial hospital. The health care system functions very efficiently too. In addition, Vietnam also has a good hospital database system which was very helpful for us.

(iv) Another advantage is health care cost for children under 6 years, which is free of charge and then access to the hospital in our area is good. Within 30 minutes, the whole area can get to their healthcare facility.

These are the advantages of our study.

\section{Disadvantages or limitations}

(i) Population movement in the study area; there is always population movement, people are moving in and out like $10 \%$ to $15 \%$ every year, so you have to regularly monitor and update the population data. Since the study area is large, it requires a huge budget for this regular update.

(ii) Community and hospital database matching; when we try to match these databases, but we can never get $100 \%$. The maximum is $70 \%$. Why? When they hospitalize, sometimes they give a different name, nickname or they give a different address like their relative's addresses rather than a correct one, so we could not link it correctly. We still we need to find way to improve this process.

(iii) Low rate of diseases observed in low social economic class; Nha Trang is a kind of middle-class socioeconomic status, there are limited number of cases related to low-socioeconomic class like severe malnutrition, severe diseases, so it is not good to study such kind of diseases in our study area.

(iv) Every year, the socioeconomic status is going up in the region and since the living cost is rising year by year, local staffs are demanding more and more sal- ary. That's also a big burden to continue to maintain this large study site.

\section{Questions AND Discussion}

Tetsu Yamashiro Thank you very much Dr. Yoshida. He has presented the different variety of research work using the same the cohort and also hospitalbased study every year. I would like to invite one question due to limitation of the time, pressing of the time, if somebody has a question? Any question? You mentioned that the outbreak of dengue is closely related to the density of the population.

Lay-Myint Yoshida That's correct.

Tetsu Yamashiro Yeah, I mean the density of the population is within some certain window.

Lay-Myint Yoshida That's right.

Tetsu Yamashiro So, I expect that if the population is more condensed, much more outbreak may occur, but it was not, do you have an explanation on it?

Lay-Myint Yoshida We didn't have the entomological data, but I guess there might be optimal ratio between the population density and mosquito surrounding them. Even if there were too many people in the area if the mosquito density was not optimal, we will not see large outbreaks.

Tetsu Yamashiro Very interesting data. Thank you very much. Okay, thank you very much.

Lay-Myint Yoshida Thank you very much.

\section{REFERENCES}

1. UNICEF. The state of world's children 2008. http:// www.unicef.org/sowc08/docs/sowc08.pdf. (http://www. who.int/mediacentre/factsheets/fs117/en/)

2. Yanai H, Thiem VD, Matsubayashi T, Huong VTT, Suzuki M, Mai LP, Anh NH, Tho LH, Minh TT, Yoshida L-M, Kilgore P, Anh DD, Ariyoshi K. The Khanh Hoa Health Project: Characterization of Study Population and Field Site Development for Clinical Epidemiological Research on Emerging and Re-Emerging Infectious Diseases. Trop Med Health 2007; 35(2): 61-63.

3. Suzuki M, Thiem VD, Yanai H, Matsubayashi T, Yoshida L-M, Tho LH, Minh TT, Anh DD, Kilgore P, Ariyoshi K. Association of enviromental tabacco smoking exposure with an increased risk of hospital admissions for pneumonia in children under 5 years of age in Vietnam. Thorax 2009; 64(6): 484-489.

4. Suzuki M, Vu TD, Yoshida L-M, Anh DD, Kilgore PE, Ariyoshi K. Who are exposed to smoke at home?-A population-based cross-sectional survey in central Vietnam. Tob Control 2010; 19(4): 344-345.

5. Tsuzuki A, Thiem DV, Suzuki M, Matsubayashi T, 
Yoshida L-M, Tho Le H, Minh TT, Anh DD, Kilgore P, Takagi M, Ariyoshi K. Can Daytime Bed-net Usage Reduce the Risk of Dengue Hemorrhagic Fever among Children in Vietnam? Am J Trop Med Hyg 2010; 82(6): 1157-1159.

6. Schmidt WP, Suzuki M, Thiem VD, Tsuzuki A, Yoshida L-M, Yanai H, Haque U, Tho LH, Anh DD, Ariyoshi K. Population density, water supply and the risk of dengue fever in Vietnam: cohort study and spatial analysis. Plos Med 2011; 8(8): e1001082.

7. Thiem VD, Schmidt W-P, Suzuki M, Tho LH, Yanai H, Ariyoshi K, Anh DD, Yoshida L-M. Animal livestock and the risk of hospitalized diarrhea in children under 5 years in Vietnam. Trop Med Int Health 2012; 17(5): 613-621.

8. Anh DD, Kilgore PE, Slack MPE, Nyambat B, Tho LH, Yoshida LM, Nguyen HA, Nguyen CD, Chong CY, Nguyen D, Ariyoshi K, Clemens JD, Jodar L. Surveillance for Pneumococcal-Associated Disease in Hospitalized Children in Khanh Hoa Province, Vietnam. Clin Infect Dis 2009; 48(Suppl 2): S57-S64.

9. Yoshida L-M, Suzuki M, Yamamoto T, Nguyen HA, Nguyen CD, Nguyen AT, Oishi K, Vu TD, Le TH, Le MQ, Yanai H, Kilgore PE, Dang DA, Ariyoshi K. Viral Pathogens Associated with Acute Respiratory Infections in Central Vietnamese Children. Pediatr Infect Dis J 2010; 29(1): 75-77.
10. Vu HT, Yoshida LM, Suzuki M, Nguyen HA, Nguyen CD, Nguyen AT, Oishi K, Yamamoto T, Watanabe K, Vu TD, Schmidt WP, Phan HT, Morimoto K, Le TH, Yanai H, Kilgore PE, Dang AD, Ariyoshi K. Association Between Nasopharyngeal Load of Streptococcus pneumoniae, Viral Coinfection, and Radiologically Confirmed Pneumonia in Vietnamese Children. Pediatr Infect Dis J 2011; 30(1): 11-18.

11. Yoshida L-M, Suzuki M, Nguyen HA, Le MN, Dinh Vu T, Yoshino H, Schmidt W-P, Nguyen TT, Le HT, Morimoto K, Moriuchi H, Dang DA, Ariyoshi K. Respiratory syncytial virus: co-infection and paediatric lower respiratory infections. Eur Respir J 2013; 42(2): 461-469.

12. Ota E, Haruna M, Suzuki M, Anh DD, Tho le H, Tam NT, Thiem VD, Anh NT, Isozaki M, Shibuya K, Ariyoshi K, Murashima S, Moriuchi H, Yanai H. Maternal body mass index and gestational weight gain and their association with perinatal outcomes in Viet Nam. Bull World Health Organ 2011; 89(2): 127-136.

13. Miyakawa M, Yoshino H, Yoshida L-M, Vynnycky E, Motomura H, Tho LH, Thiem VD, Ariyoshi K, Anh DD, Moriuchi H. Seroprevalence of rubella in the cord blood of pregnant women and congenital rubella incidence in Nha Trang, Vietnam. Vaccine 2013. Sep 7. pii: S0264-410X(13)01188-2. doi:10.1016/j.vaccine.2013.08. 076 